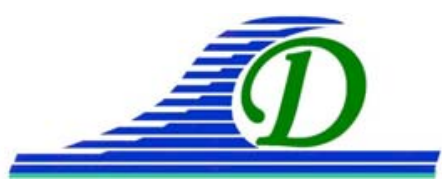

XIII ${ }^{\text {ìmes }}$ Journées Nationales Génie Côtier - Génie Civil

Dunkerque, 2-4 juillet 2014

DOI:10.5150/jngcgc.2014.051 @ Editions Paralia CFL

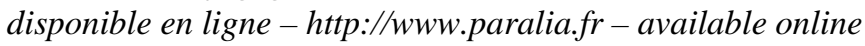

\title{
Étude de l'impact de la résolution horizontale sur la modélisation numérique de processus hydrodynamiques côtiers et des flux sédimentaires associés
}

\author{
Baptiste MENGUAL ${ }^{1}$, Florence CAYOCCA ${ }^{1}$, Franck DUMAS ${ }^{1}$, \\ Pierre LE HIR ${ }^{1}$, Thierry GARLAN ${ }^{2}$
}

1. IFREMER - DYNECO/PHYSED, Centre de Brest, BP 70, 29280 Plouzané, France. baptiste.mengual@ifremer.fr

2. SHOM, Centre militaire d’océanographie, section recherche, 29603 Brest, France.

\section{Résumé :}

Dans un modèle numérique, le choix de la résolution horizontale de la grille de calcul détermine l'échelle des processus physiques qui seront correctement représentés. Cette résolution influence par ailleurs la précision de la représentation des forçages, notamment, pour un modèle hydrosédimentaire, la contrainte de cisaillement sur le fond (moteur du processus d'érosion), et plus généralement la circulation hydrodynamique. La sensibilité à la résolution est particulièrement élevée dans les zones côtières présentant de forts gradients bathymétriques et de faibles profondeurs d'eau.

Cette étude s'applique à comparer les forçages, les circulations et les flux sédimentaires issus de deux simulations faites sur une même zone, pour deux périodes contrastées et pour deux résolutions différentes de $2500 \mathrm{~m}$ et $500 \mathrm{~m}$. En période de tempête, des différences significatives sur l'estimation de la contrainte de cisaillement induite par les vagues ont été mises en évidence avec des différences moyennes allant jusqu'à $40 \%$ pour des profondeurs inférieures à $20 \mathrm{~m}$. Les flux sédimentaires calculés sur les deux maillages ont été comparés le long de différents transects caractérisés par différentes profondeurs d'eau. La haute résolution permet la représentation de structures de circulation particulières sur tout le domaine, et des différences significatives de concentrations en matières en suspension ont été observées le long de ces transects sur l'ensemble de la colonne d'eau, en particulier pour les sédiments vaseux.

L'ensemble de ces résultats apporte des éléments de réponse sur l'influence de la résolution sur la quantification des flux sédimentaires.

Mots clés : Résolution horizontale, Forçages hydrodynamiques, Flux sédimentaires, Modélisation numérique.

\section{Introduction}

Les mouvements sédimentaires en milieu marin résultent de facteurs dynamiques naturels ou anthropiques, tels que la remise en suspension, le dépôt et le transport de sédiments sous l'effet des courants et des vagues. Ces mouvements peuvent se traduire par des évolutions morphologiques et par des modifications de la nature des fonds. 


\section{Thème 2 - Dynamique sédimentaire}

La représentation de ces phénomènes par un modèle numérique dépend en particulier de sa capacité à estimer correctement les différents processus hydrodynamiques. En particulier en domaine côtier, cette capacité est notamment liée au choix de la résolution horizontale qui détermine l'échelle spatiale des structures pouvant être mises en évidence (GREENBERG et al., 2007). Par ailleurs, seule une représentation raffinée du trait de côte et de la bathymétrie (par exemple des chenaux) permet de reproduire correctement certains écoulements côtiers comme dans le cas de détroits, d'embouchures, ou encore de pertuis (MOLINES et al., 1989 ; GILLE et al., 2004). Une grille non-structurée ou plusieurs grilles structurées emboitées constituent des solutions à ces types de problèmes (MOLINES et al., 1989 ; LAVAL et al., 2003).

Ce travail s'applique à répondre à la question suivante : dans quelle mesure les flux régionaux fournis par une configuration discrétisant le Golfe de Gascogne (Côte Atlantique Française) à une résolution à 2500 mètres sont-ils améliorés par l'utilisation d'un zoom côtier de résolution plus fine (500 mètres de résolution). En d'autres termes, est ce qu'un raffinement côtier aboutit à la mise en évidence de différences significatives sur la qualité de la représentation des forçages, sur la circulation hydrodynamique, et donc sur les flux sédimentaires associés ? En effet, les différences induites par l'augmentation de la résolution près des côtes sont susceptibles de modifier les flux résiduels vers le large ou dans les domaines plus profonds, et influencer ainsi les flux à l'échelle régionale. Ce travail propose de premières réponses à ces questions, afin de déterminer la stratégie optimale pour la détermination des flux sédimentaires à différentes échelles spatiales.

\section{Matériels et Méthodes}

\subsection{Modèle utilisé}

Le modèle utilisé dans le cadre de cette étude est le modèle hydrodynamique MARS3D développé par l'Ifremer. Il résout les équations de Navier-Stokes pour un fluide supposé incompressible, hydrostatique et suivant l'hypothèse de Boussinesq, par une méthode de différences finies sur des maillages structurés. Ces équations expriment en chaque point du domaine de calcul la conservation de la masse et de la quantité de mouvement dans les trois directions de l'espace. Ce modèle permet de calculer les courants et résiduelles eulériennes, les hauteurs d'eau, la température, la salinité, ou encore le transport de particules en suspension. MARS3D utilise une technique d'emboîtement de modèles sous forme gigogne, où une configuration de grande emprise et de faible résolution fournit les conditions aux limites de sous modèles de plus haute résolution. Deux configurations de résolutions différentes, à $2500 \mathrm{~m}$ et $500 \mathrm{~m}$, ont été utilisées dans le cadre de cette étude comparative (figure 1). La discrétisation verticale est quant à elle commune aux deux configurations avec 40 niveaux sigma. 


\section{XIII ${ }^{\text {èmes }}$ Journées Nationales Génie Côtier - Génie Civil \\ Dunkerque, 2-4 juillet 2014}
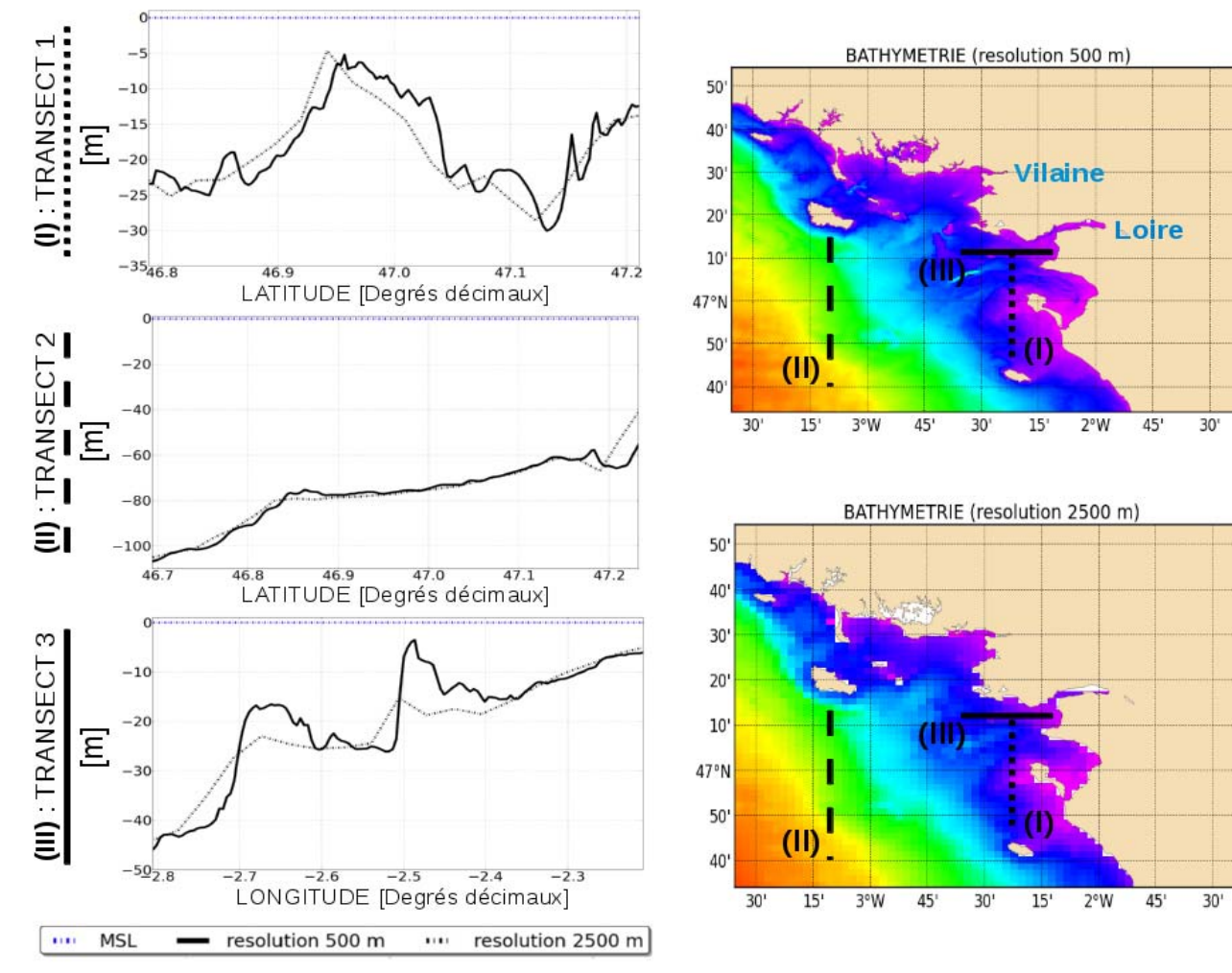

M / MSL

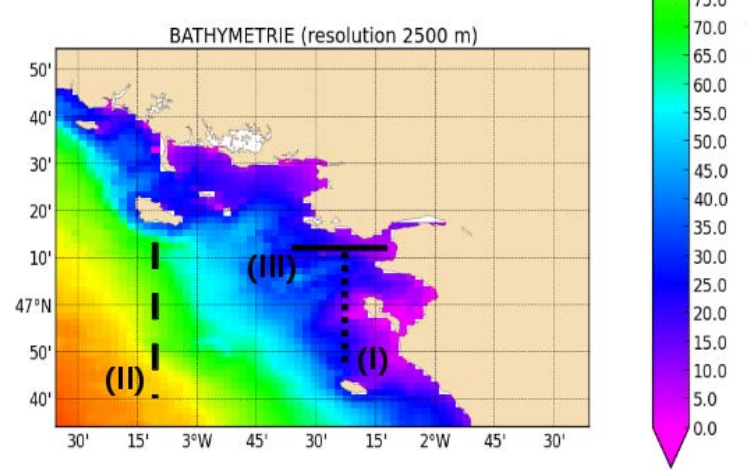

Figure 1. Représentation des bathymétries à 500 et $2500 \mathrm{~m}$ de résolution et des différents transects par rapport au niveau moyen (MSL).

La configuration à $2500 \mathrm{~m}$ de résolution, initialement forcée aux limites par une emprise plus large, a été utilisée afin d'extraire les conditions aux limites et la condition initiale de la configuration à $500 \mathrm{~m}$ de résolution. Les deux configurations sont forcées par le modèle Arpege haute résolution (i.e. $10 \mathrm{~km}$ ) pour le vent. Une configuration côtière non structurée du modèle WAVEWATCH III ${ }^{\circledR}$ (RASCLE \& ARDHUIN, 2013) fournit les contraintes de cisaillement liées aux vagues; ces contraintes sont ensuite projetées sur les deux grilles. Le module sédimentaire décrit dans LE HIR et al. (2011) a par ailleurs été utilisé avec trois classes de sédiments caractérisées par un sable fin (MES1), un sédiment vaseux (MES2) et une vase à faible vitesse de chute (MES3) associée aux apports fluviaux (la Loire et la Vilaine). Afin de réduire les temps de calcul imposés par les vitesses de chute élevées pour le sable, le transport des MES1 dans la colonne d'eau est fait de manière intégrée sur la verticale ; l'hypothèse d'un profil de Rouse pour le sable permet ensuite de calculer des flux horizontaux sur la colonne d'eau prenant en compte l'existence de forts gradients verticaux de concentration (WAELES et al., 2007). Une même description de la composition initiale du fond (proportions différentes des trois classes de sédiments permettant de représenter différents faciès réalistes) a été utilisée pour les deux grilles. 


\section{Thème 2 - Dynamique sédimentaire}

\subsection{Stratégie}

Trois transects ont servi de base pour estimer l'influence de la résolution horizontale sur les flux (figure 1). Les transects 1 et 3 situés à l'embouchure de la Loire ont pour but de comparer les dynamiques liées aux processus hydrodynamiques, hydrologiques, et sédimentaires pour des profondeurs d'eau inférieures à $50 \mathrm{~m}$. Le transect 2 est situé plus au large par des profondeurs proches de la centaine de mètres; ce choix permet d'examiner l'influence de la résolution sur les flux vers le large, dans une zone de faibles gradients bathymétriques (et par conséquent, de faibles gradients de forçage). L'influence de la résolution horizontale est étudiée pour deux périodes de conditions hydro-météorologiques contrastées. Les deux simulations ont débuté 15 jours avant chaque période d'intérêt. La première période, qualifiée de faiblement énergétique, s'étend du $1^{\mathrm{er}}$ au 3 décembre 2013 et est caractérisée par une houle de secteur Ouest/Sud-Ouest de $1 \mathrm{~m}$ de hauteur significative, de $5 \mathrm{~s}$ de période de pic au niveau de la frontière Ouest du domaine, par des vents d'Est à Nord-Est faibles de quelques mètres par secondes et des débits fluviaux moyens (Loire : $1120 \mathrm{~m}^{3} \mathrm{~s}^{-1}$; Vilaine : $45 \mathrm{~m}^{3} \mathrm{~s}^{-1}$ ). La seconde période, du 6 au 11 janvier 2014, s'inscrit dans un contexte très énergétique faisant suite au passage de la tempête Petra. La houle au large de secteur Ouest/SudOuest atteint des hauteurs significatives de plus de $5 \mathrm{~m}$ le 7 janvier et des périodes de 10 à $12 \mathrm{~s}$ au niveau de la frontière Ouest, associées à des vents de secteur Ouest/Sud-Ouest à Sud/Sud-Est de $15 \mathrm{~m} . \mathrm{s}^{-1}$, et des débits fluviaux importants (Loire: $1900 \mathrm{~m}^{3} \mathrm{~s}^{-1}$; Vilaine : $285 \mathrm{~m}^{3} \mathrm{~s}^{-1}$ ) traduisant d'importantes crues liées aux fortes précipitations.

\section{Résultats}

\subsection{Impact de la résolution sur la circulation hydrodynamique}

Des différences significatives ont pu être observées sur la circulation hydrodynamique d'une résolution à l'autre. La configuration à $2500 \mathrm{~m}$ de résolution présente des fronts de densité très marqués sur le plateau (au-delà de $50 \mathrm{~m}$ de fond), qui semblent déstabilisés dans la configuration à $500 \mathrm{~m}$ (apparition de structures de méso-échelle cisaillées). Afin d'examiner le réalisme de ces structures, trois valeurs $(0.1,0.2$, et 0.3$)$ du coefficient de Smagorinsky (SMAGORINSKY, 1973) relatif à la paramétrisation de la viscosité turbulente horizontale ont été testées, conformément à sa plage de variation (entre 0.05 et 0.3 ; cf. HOLT \& JAMES, 2006). Ces structures cisaillées persistent même pour des valeurs élevées de viscosité turbulente horizontale (SMAGORINSKY, 1973), ce qui conforte l'hypothèse de vraisemblance de cette circulation. La haute résolution représente ainsi des gradients importants de densité sur de faibles distances (quelques centaines de mètres à quelques kilomètres) qui influencent directement les vitesses, et plus généralement la circulation hydrodynamique. 


\section{XIII ${ }^{\text {èmes }}$ Journées Nationales Génie Côtier - Génie Civil \\ Dunkerque, 2-4 juillet 2014}

\subsection{Impact de la résolution sur les contraintes de cisaillement}

L'impact de la résolution sur l'estimation de la contrainte de cisaillement sur le fond $\tau$, moteur du processus érosif, a tout d'abord été étudié. Des moyennes par strates bathymétriques ont été réalisées par tranches de 5-10 m, 10-20 m, 20-40 m, 40-60 m, 60 $-80 \mathrm{~m}$, et $80-100 \mathrm{~m}$.

Durant la période calme, les ordres de grandeur de $\tau$ sont globalement faibles avec des valeurs moyennées par strate bathymétrique de 0.4 à $0.6 \mathrm{~N} \mathrm{~m}^{-2}$ au plus (valeurs maximales atteintes près de la côte). Les différences les plus significatives d'une résolution à l'autre interviennent sur les contraintes de cisaillement liées au courant $\tau_{c}$ entre 5 et $10 \mathrm{~m}$ de fond, avec des différences ponctuelles d'un facteur 4 résultant de la surestimation de $\tau_{c}$ dans la configuration grossière (où ces zones peu profondes sont mal représentées). Au-delà de $20 \mathrm{~m}$ de fond, les contraintes $\tau_{c}$ sont équivalentes d'une résolution à l'autre et contribuent à $50 \%$ de la contrainte totale. Les différences d'une configuration à l'autre sur les valeurs de contraintes liées aux vagues $\tau_{w}$ ne sont pas significatives (0.05 $\mathrm{N} \mathrm{m}^{-2}$ au maximum). La contribution des vagues à la contrainte totale est dominante pour des profondeurs d'eau inférieures à $20 \mathrm{~m}$ avec des valeurs 2 à 3 fois plus importantes que celles relatives à l'influence du courant. Au-delà, elle devient équivalente à celle liée au courant.

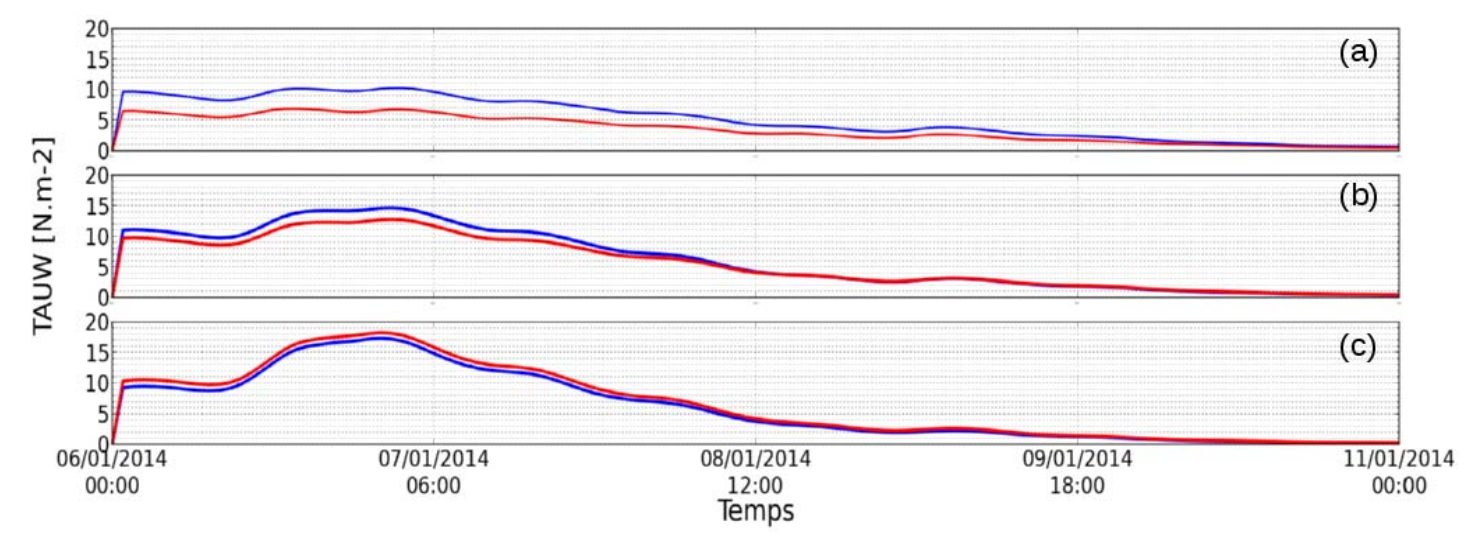

Figure 2. Moyennes de la contrainte de cisaillement induite par les vagues (TAUW) par strate bathymétrique $[(a): 5-10 \mathrm{~m} ;(b): 10-20 \mathrm{~m} ;(c): 20-40 \mathrm{~m}]$ pour les configurations à $500 \mathrm{~m}$ (bleu) et $2500 \mathrm{~m}$ (rouge) de résolution durant la période énergétique.

Durant la période de tempête de début janvier, la contribution des vagues à la contrainte totale est dominante à toutes les profondeurs. D'une résolution à l'autre, les écarts les plus significatifs sont observés pour les plus faibles profondeurs d'eau, entre 5 et $20 \mathrm{~m}$. La présence de hauts fonds entre 20 et $40 \mathrm{~m}$ engendre une dissipation de l'énergie des vagues expliquant les valeurs plus importantes de $\tau_{w}$ pour cette strate bathymétrique comparativement à celles obtenues pour de plus faibles profondeurs. Le pic de houle 


\section{Thème 2 - Dynamique sédimentaire}

atteint le 7 janvier à $00 \mathrm{~h} 00$ coïncide avec les écarts les plus importants sur $\tau_{w}$, avec une sous-estimation sur la grille grossière de $40 \%$ entre 5 et $10 \mathrm{~m}$, et $15 \%$ entre 10 et $20 \mathrm{~m}$ par rapport à la grille de haute résolution (figure 2). Par ailleurs, il est important de souligner les valeurs exceptionnelles de ces contraintes avec près de $18 \mathrm{~N} \mathrm{~m}^{-2}$, soit environ 30 fois plus que les valeurs observées durant la première période. Au-delà de $20 \mathrm{~m}$ de fond, mises à part de légères différences pendant le début de période très énergétique, les contraintes sont proches sur les deux grilles.

\subsection{Impact de la résolution sur les flux sédimentaires}

Les flux sédimentaires ont été étudiés le long de chaque transect, pour chacune des deux périodes et pour les deux grilles.

Durant la période de tempête de début janvier 2014, les coupes le long des transects 1 (figure 3) et 2 (figure 4) ont été représentées lors du pic de houle le 07 à $00 \mathrm{~h} 00$.
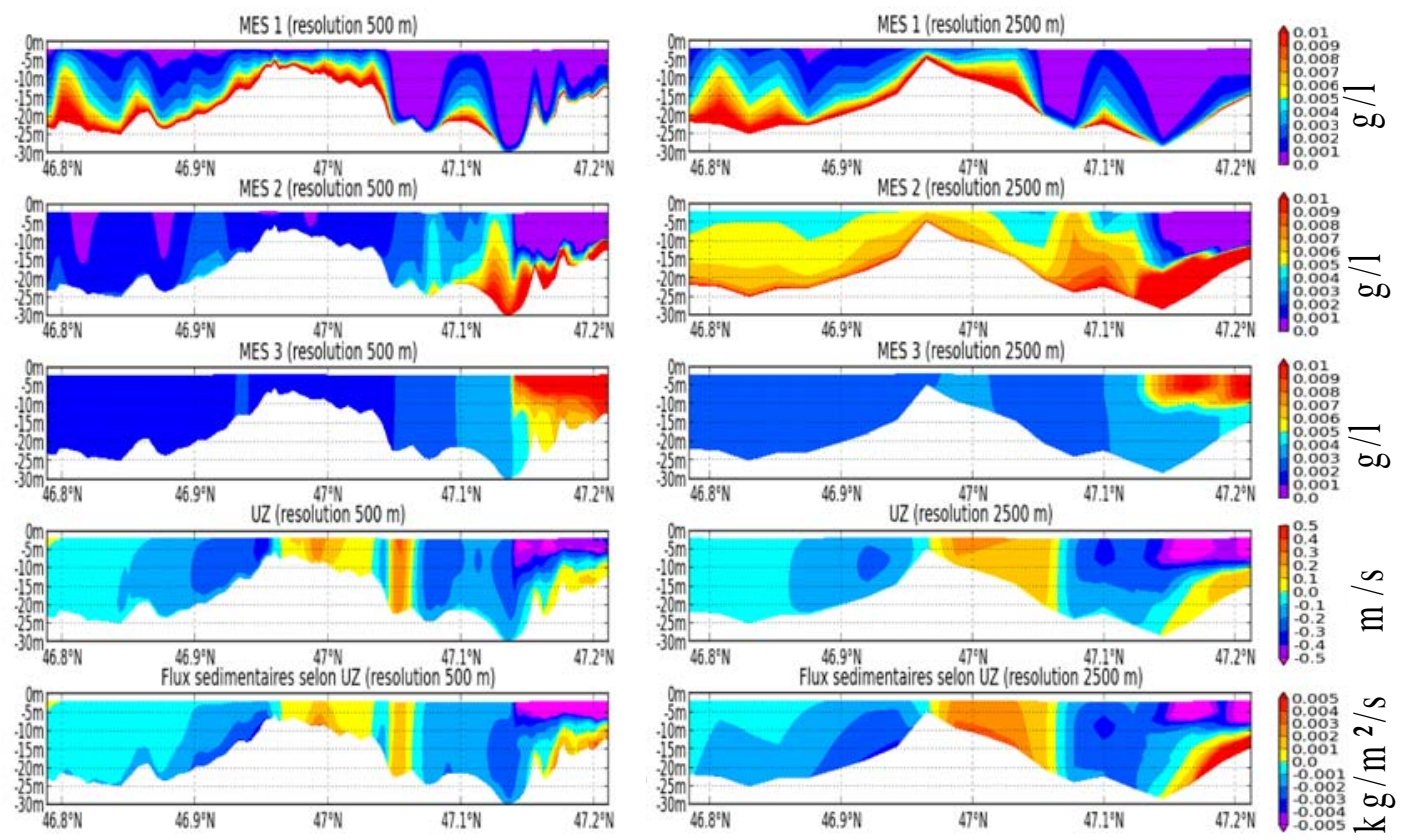

Figure 3. Coupes des concentrations en MES ( $\mathrm{g} / \mathrm{l})$, de la composante U (Est-Ouest positive vers l'Est) des vitesses de courant ( $\mathrm{m} / \mathrm{s})$, et des flux sédimentaires $\left(\mathrm{kg} \mathrm{m}^{-2} \mathrm{~s}^{-1}\right)$ le long du transect 1 lors de la période énergétique (7 janvier 2014 à 00h), pour les deux résolutions.

Sur la figure 3, on peut tout d'abord justifier les allures semblables des profils verticaux de concentrations en sable à l'échelle de la colonne d'eau du fait qu'elles ont été reconstituées selon un profil de Rouse à partir de la concentration intégrée sur la verticale. On peut cependant noter des différences significatives sur les MES2 et MES3. 


\section{XIII ${ }^{\text {èmes }}$ Journées Nationales Génie Côtier - Génie Civil \\ Dunkerque, 2-4 juillet 2014}

Pour les MES2, les concentrations en particulier en début de profil sont nettement plus importantes dans la configuration basse résolution sur l'ensemble de la colonne d'eau avec des valeurs 10 fois plus importantes près du fond. Ces différences ne peuvent être imputées aux seules différences de contraintes entre les deux résolutions, elles résultent donc de la représentation différente de l'advection sur les deux grilles. Pour les MES3, on peut observer une stratification du panache de la Loire bien plus marquée sur la configuration à faible résolution. La combinaison des différences de concentrations et de vitesses explique les différences observées sur les flux sédimentaires.
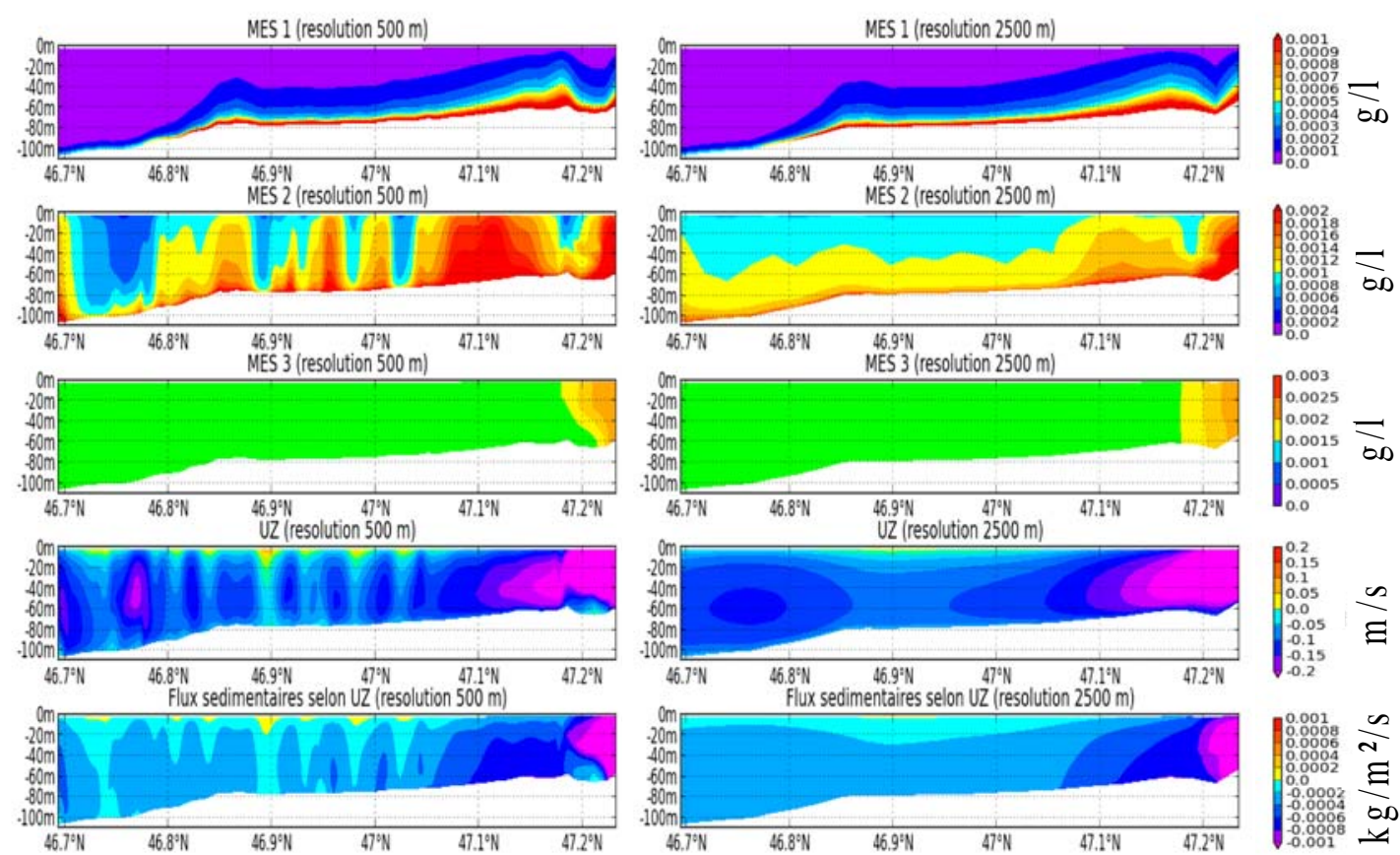

Figure 4. Coupes des concentrations en MES ( $\mathrm{g} / \mathrm{l})$, de la composante U (Est-Ouest positive vers l'Est) des vitesses de courant ( $\mathrm{m} / \mathrm{s})$, et des flux sédimentaires $\left(\mathrm{kg} \mathrm{m}^{-2} \mathrm{~s}^{-1}\right)$ le long du transect 2 lors de la période énergétique (7 janvier 2014 à 00h), pour les deux résolutions.

La figure 4 représente le transect 2. On peut tout d'abord y observer des concentrations globalement 10 fois plus faibles que sur le transect 1 , et des vitesses de courant moins importantes. Pour la MES2, bien que les gammes de concentrations soient proches pour les deux résolutions, la répartition des sédiments en suspension à l'échelle de la colonne d'eau est très contrastée d'une configuration à l'autre. En effet, dans la configuration à haute résolution, les MES2 sont plus concentrées sur la majeure partie du transect et remontent localement sur l'ensemble de la colonne d'eau. Ce phénomène n'est pas observé dans le cas d'une résolution plus grossière avec des concentrations plus faibles et peu de diffusion verticale des fines. De plus, la configuration haute résolution 


\section{Thème 2 - Dynamique sédimentaire}

représente des profils de vitesses cisaillés non visibles à basse résolution. Cette circulation particulière combinée aux différences de concentration est à l'origine des différences de flux sédimentaires observées.

Les résultats relatifs à la période calme (non illustrés ici) ont montré au niveau du transect 1 des concentrations globalement 10 fois plus faibles qu'au cours de la période énergétique. La configuration à $500 \mathrm{~m}$ a mis en évidence des concentrations plus importantes en MES2 et MES3 qu'à faible résolution, avec des différences locales instantanées de près de $50 \%$. De même, la diffusion des matières en suspension sur la verticale est plus intense à haute résolution. Les différences de concentrations associées à des vitesses légèrement plus importantes pour la configuration haute résolution ont permis d'expliquer localement les différences sur les flux sédimentaires. Les résultats obtenus le long du transect 2 (au large) ont montré l'absence de MES1 et MES2 en suspension avec des flux sédimentaires faibles uniquement liés aux concentrations résiduelles en MES3.

Les résultats relatifs au transect 3 ont permis de mettre en évidence une dispersion plus importante du panache de la Loire, tant sur l'horizontal que sur la verticale, dans la configuration à haute résolution pour les deux périodes.

\section{Discussion et conclusions}

Les deux périodes contrastées d'un point de vue énergétique ont permis de comparer l'influence de la résolution sur la contrainte de cisaillement sur le fond, résultant de l'action combinée des vagues et des courants. En conditions peu énergétiques, les contraintes de cisaillement sur le fond sont faibles $\left(<1 \mathrm{~N} \mathrm{~m}^{-2}\right)$ et globalement proches d'une résolution à l'autre. En revanche, pour des conditions très énergétiques, l'influence de la résolution est importante pour des profondeurs d'eau inférieures à $20 \mathrm{~m}$, notamment sur l'estimation de la contrainte de cisaillement induite par les vagues, avec des valeurs moyennes jusqu'à $40 \%$ plus importantes pour la configuration à $500 \mathrm{~m}$ de résolution.

Le long des transects 1 et 2, pour des contraintes de cisaillement proches, des différences significatives sur les concentrations en MES2 (vase) d'une configuration à l'autre ont démontré l'influence des processus d'advection dans l'estimation à un instant donné de ces concentrations, qui se traduisent sur les flux sédimentaires résultants. De plus, l'amélioration de la représentation tridimensionnelle de la dynamique des panaches fluviaux (en l'occurrence la Loire) avec la résolution a été observée grâce aux profils de concentration et flux associés à la MES3. Plus au large, le long du transect 2, des cellules de circulation complexes ont été mises en évidence uniquement avec la résolution à $500 \mathrm{~m}$. On peut penser que la similitude des concentrations en MES1 obtenues le long des deux transects pour les deux résolutions est liée d'une part au calcul intégré sur la verticale, d'autre part à une faible sensibilité des concentrations de sable à l'advection du fait de sa vitesse de chute élevée. Toutefois, une modification de 


\section{XIII ${ }^{\text {èmes }}$ Journées Nationales Génie Côtier - Génie Civil \\ Dunkerque, 2-4 juillet 2014}

la discrétisation verticale permettrait probablement de mettre en évidence des différences de résultats d'une résolution à l'autre (GREENBERG et al., 2007). Les résultats relatifs à la période faiblement énergétique ont mis évidence des concentrations en MES, des vitesses de courant, et donc des flux sédimentaires largement plus faibles. Ils ont montré qu'en l'absence d'un forçage par les vagues, seuls les sédiments les plus fins (MES3) sont en suspension au large (à $80 \mathrm{~m}$ de fond).

Cette étude a donc permis d'apporter des premières réponses quant à l'influence de la résolution horizontale sur la quantification de la concentration en matières en suspension, la circulation et les flux sédimentaires associés, en se plaçant dans deux contextes énergétiques particuliers. Cependant, il serait nécessaire de réaliser ces comparaisons à plus longue échelle de temps, par exemple pendant plusieurs cycles de morte eau / vive eau, et de mieux comprendre l'influence de chaque forçage (courants, vagues) sur les flux sédimentaires instantanés. Des comparaisons faites sur les courants résiduels d'une résolution à l'autre permettraient de dissocier la part des différences liée aux processus d'advection de celle liée à la meilleure représentation des forçages. Plus généralement, une meilleure analyse de la sensibilité de la circulation hydrodynamique à la résolution horizontale apporterait de nouvelles conclusions quant aux différences liées aux processus d'advection et au mélange. De même, l'étude de l'évolution de la nature sédimentaire du fond ajouterait un point important dans l'interprétation des résultats. Enfin, des comparaisons pour de plus hautes résolutions et sur d'autres zones complémentaires permettraient de vérifier et compléter les résultats de cette étude, et notamment d'examiner dans quelle mesure il est intéressant de raffiner la résolution à la côte en fonction des échelles et processus considérés.

\section{Remerciements}

Cette étude a été cofinancée par le SHOM (Service Hydrographique et Océanographique de la Marine) et par IFREMER (Institut Français de Recherche pour l’Exploitation de la Mer).

\section{Références bibliographiques}

GILLE S.T., METZGER E.J., TOKMAKIAN R. (2004). Seafloor topography and ocean circulation. Oceanography, Vol. 17(1), pp 47-54. http://dx.doi.org/10.5670/oceanog.2004.66

GREENBERG D.A., DUPONT F., LYARD F.H., LYNCH D.R., WERNER F.E. (2007). Resolution issues in numerical models of oceanic and coastal circulation. Contonental Shelf Research, Vol. 27(9), pp 1317-1343. http://dx.doi.org/10.1016/j.csr.2007.01.023 HOLT J., JAMES I. (2006). An assessment of the fine-scale eddies in a high-resolution model of the shelf seas west of Great Britain. Ocean Modelling, Vol. 13(3-4), pp 271-291. http://dx.doi.org/10.1016/j.ocemod.2006.02.005 
LAVAL B., IMBERGER J., FINDIKAKIS A.N. (2003). Mass transport between a semi-enclosed basin and the ocean: Lake Maracaibo. Journal of Geophysical Research, Vol. 108(C7), 3234. http://dx.doi.org/10.1029/2002JC001571

LE HIR P., CAYOCCA F., WAELES B. (2011). Dynamics of sand and mud mixtures: a multiprocess-based modelling strategy. Continental Shelf Research, Vol. 31(10), pp 135-149. http://dx.doi.org/10.1016/j.csr.2010.12.009

MOLINES J., FORNERINO M., LE PROVOST C. (1989). Tidal spectro-scopy of a coastal area: observed and simulated tides of the Lake Maracaibo system. Continental Shelf Research, Vol. 9(4), pp 301-323. http://dx.doi.org/10.1016/0278-4343(89)90036-8

RASCLE N., ARDHUIN F. (2013). A global wave parameter database for geophysical applications. Part 2: Model validation with improved source term parameterization. Ocean Modelling, Vol. 70, pp 174-188. http://dx.doi.org/10.1016/j.ocemod.2012.12.001

SMAGORINSKY J. (1963). General circulation experiments with the primitive equations. Monthly Weather Review, Vol. 91, pp 99-164. http://dx.doi.org/10.1175/15200493(1963)091<0099:GCEWTP>2.3.CO;2

WAELES B., LESUEUR P., LE HIR P. (2007). Modelling sand/mud transport and morphodynamics in the Seine river mouth (France) : an attempt using a process-based approach. Hydrobiologia, Vol. 588, pp 69-82. http://dx.doi.org/10.1007/s10750-007-0653-2 